\title{
Necrotizing Fasciitis from a Percutaneous Transhepatic Biliary Drainage Site
}

\author{
Jangyoun Choi, Jin Tae Cho, Jong Yun Choi, Sung-No Jung, Bommie Florence Seo \\ Department of Plastic and Reconstructive Surgery, College of Medicine, The Catholic University of Korea, Seoul, Korea
}

\begin{abstract}
Percutaneous transhepatic biliary drainage (PTBD) is the established method of treatment for benign or malignant biliary obstruction. Although relatively safe, the procedure is associated with complications including intraperitoneal bile leak, pancreatitis, cholangitis, biliary peritonitis, and hemorrhage. Extraperitoneal complications are rare. Here, we document a case in which necrotizing fasciitis developed in the extraperitoneal plane surrounding the transcutaneous drainage site. The patient was managed with PTBD removal, drainage and debridement of necrotic tissues, and intravenous antibiotics.
\end{abstract}

Keywords: Fasciitis, necrotizing; Biliary tract diseases; Debridement

\section{Introduction}

Percutaneous transhepatic biliary drainage (PTBD) is one of the two standardized drainage methods for benign or malignant biliary obstruction, the other being endoscopic retrograde biliary drainage [1,2]. PTBD requires inserting a catheter in the biliary duct through a percutaneous incision either on the right side or left side of the abdomen. The procedure is performed under visualization using fluoroscopic assistance or ultrasound guidance.

Although uncommon, there have been reports of complications including sepsis, bleeding, intraperitoneal abscesses, and peritonitis in around 2\% of cholangiographies, and up to $8 \%$ of therapeutic interventions [3]. Most major, life-threatening complications are related to poor visualization of vessels during puncture, while minor complications stem from dislocation or dislodgement of the drain. Though there have been several cases of intraperitoneal abscesses, extraperitoneal complications are relatively rare.

Necrotizing fasciitis is an uncommon, rapidly progressing and fatal infection of the subcutaneous and fascial regions which can cause death. This infection may begin from a simple external laceration or a perforated appendicitis [4]. The disease is characteristically fulminant and requires emergent surgical intervention and cautious application of intravenous antibiotics. This is a case report of a patient who sustained necrotizing fasciitis of the extraperitoneal fascia surrounding the PTBD site. The study was performed in accordance with the principles of the Declaration of Helsinki. Written informed consent was obtained from the patient for the use of her images.

\section{Case}

A 64-year-old female patient with underlying diabetes mellitus, hypertension, and

\section{Case Report}

Received: August 1, 2019

Revised: August 26, 2019

Accepted: August 27, 2019

\section{Corresponding author: \\ Bommie Florence Seo, M.D., Ph.D.}

Department of Plastic and Reconstructive Surgery, Uijeongbu St. Mary's Hospital, College of Medicine, The Catholic University of Korea, 271 Cheonbo-ro, Uijeongbu 11765, Korea Tel: +82-31-820-3074

Fax: +82-31-874-0301

E-mail: bommiefseo@gmail.com

This is an Open Access article distributed under the terms of the Creative Commons Attribution Non-Commercial License (http://creativecommons.org/licenses/by-nc/4.0/) which permits unrestricted non-commercial use, distribution, and reproduction in any medium, provided the original work is properly cited.

C) 2019 Korean Wound Management Society 
end stage renal disease (ESRD) for which she was undergoing hemodialysis three times a week, was sent for consultation to the plastic surgery department for an acute infection in her right flank area. She had been diagnosed with cholangitis by magnetic resonance cholangiopancreatography, and had been

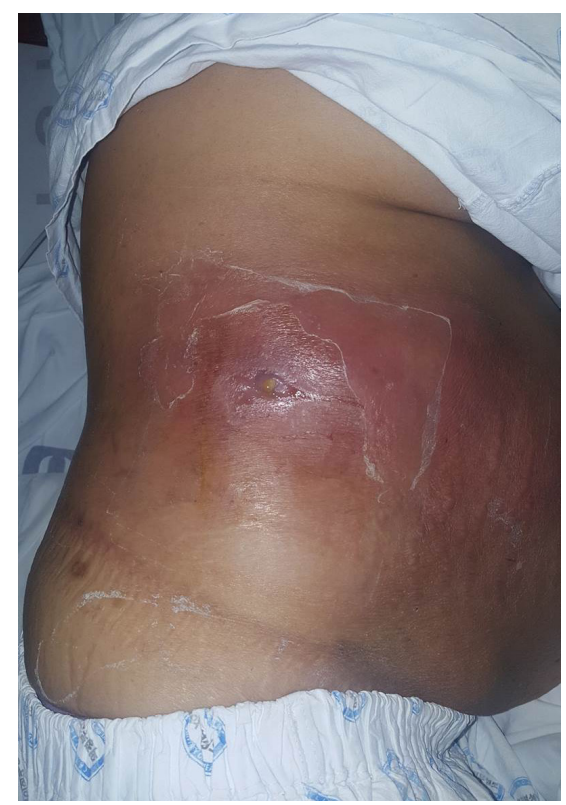

Fig. 1. Clinical image of initial patient presentation. Extensive inflammatory signs including erythema, bulging, and discharge can be seen on the patient's percutaneous transhepatic biliary drainage insertion site on the right flank.

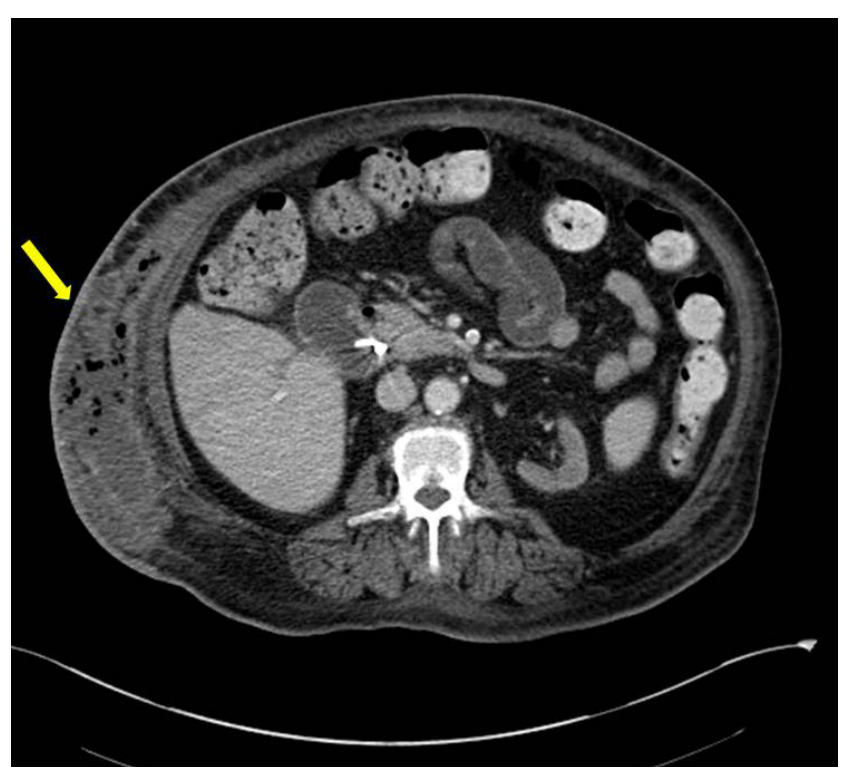

Fig. 2. Computed tomography taken on initial presentation. Extensive involvement of the fascia along the abdominal musculature, abscess formation, and emphysema are clearly visible (arrow). receiving biliary drainage via PTBD for 7 days after which the infection signs were noticed. Three years prior, the patient had been diagnosed with stones in the common bile duct and cholecystitis which had been treated with endoscopic retrograde cholangiopancreatography (ERCP) and laparoscopic cholecystectomy. This time, an attempt at ERCP had failed on account of severe adhesions caused by the previous abdominal surgery and ERCP. PTBD was performed immediately after the failure of ERCP. Immediately after PTBD insertion, biliary drainage of over 1,000 mL/day was noted. However, 1 week after drainage, upon presentation to the plastic surgery department, she had developed a fever over $38^{\circ} \mathrm{C}$ and erythematous changes around the PTBD site (Fig. 1).

Computed topography (CT) scans revealed edematous swelling with fat infiltration and air collection in the extraperitoneal muscular layer of the right flank (Fig. 2). Evaluation of the LRINEC (Laboratory Risk Indicator for Necrotizing Fasciitis) score implied a high risk of necrotizing fasciitis (Table 1) [5]. PTBD was removed 10 days after insertion by radiologist and surgical debridement was performed under general anesthesia on following day. Extensive necrosis of the subcutaneous fat layer, muscular fascia, and the external oblique and internal oblique muscle was accompanied by a foul odor and pus drainage (Fig. 3). Histopathologic findings correlated with characteristics of necrotizing fasciitis by mixed infection (Fig. 4). Wound culture studies revealed a mixed infection by Enterococcus faecalis, Klebsiella pneumoniae, and Candida species (Candida glabrata) which correspond with the most common pathogens causing cholangitis [6]. Intravenous antibiotics were changed from Tazoperan (piperacillin sodium $2 \mathrm{~g}$, tazobactam 0.25 g; Daewoong Pharm, Seoul, Korea) to Meropenem (Meropenem 500 mg; Yuhan, Seoul, Korea), Vancomycin (vancomycin $\mathrm{HCl} 1 \mathrm{~g}$; CJ Healthcare, Seoul, Korea), and Micafungin (micafungin sodium $50 \mathrm{mg}$; Astellas Pharm,

Table 1. Accessed LRINEC score on the patient

\begin{tabular}{lcc}
\hline Variable & Value & Accessed LRINEC score \\
\hline C-reactive protein $(\mathrm{mg} / \mathrm{L})$ & 181 & 4 \\
Total white blood cell count $\left(\times 10^{3} / \mathrm{\mu L}\right)$ & 17.6 & 1 \\
Hemoglobin $(\mathrm{g} / \mathrm{dL})$ & 9.4 & 2 \\
Sodium $(\mathrm{mEq} / \mathrm{L})$ & 130 & 2 \\
Creatinine $(\mathrm{mg} / \mathrm{dL})$ & 7.38 & 2 \\
Glucose $(\mathrm{mg} / \mathrm{dL})$ & 163 & 0 \\
Total score & & 11
\end{tabular}

LRINEC, Laboratory Risk Indicator for Necrotizing Fasciitis. 


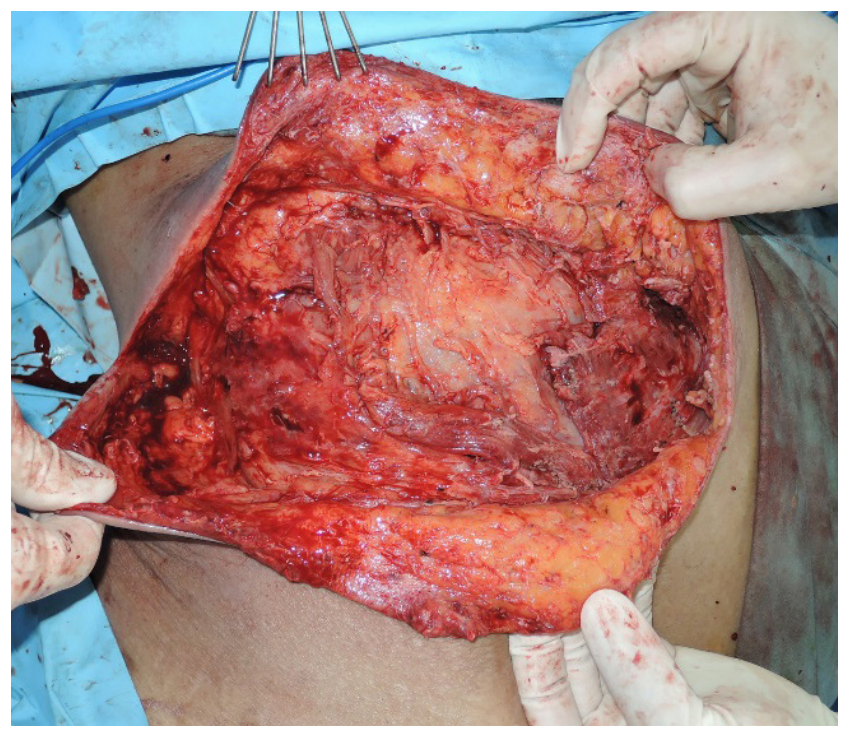

Fig. 3. Intraoperative findings of surgical debridement. Internal oblique muscle fascia was exposed after debridement of the infected external oblique muscle.

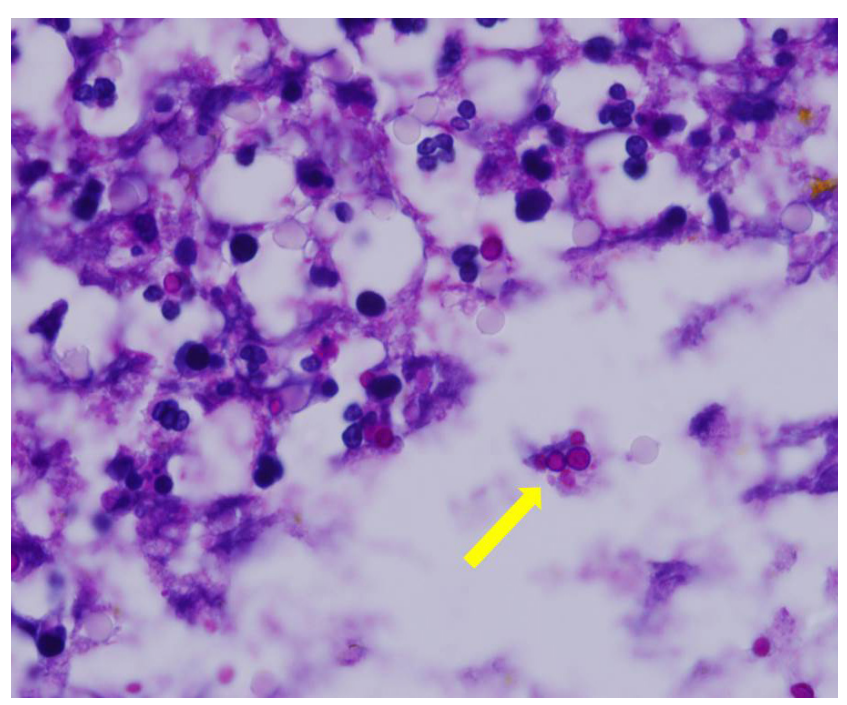

Fig. 4. Histopathology of intraoperatively obtained specimen. Histopathologic findings (periodic acid-Schiff stain, $\times 1,000$ ) showed lymphocyte infiltration of subcutaneous tissue with scattered fungus spores (arrow).

Seoul, Korea), after observing the wound culture results. After sequential debridement for 2 weeks all signs of clinical infection resolved, and the patient's general condition and cholangitis improved. A fasciocutaneous flap coverage was performed, and the wounds healed well without recurrent infection (Fig. 5). During 12 months follow-up, no additional complications were encountered.

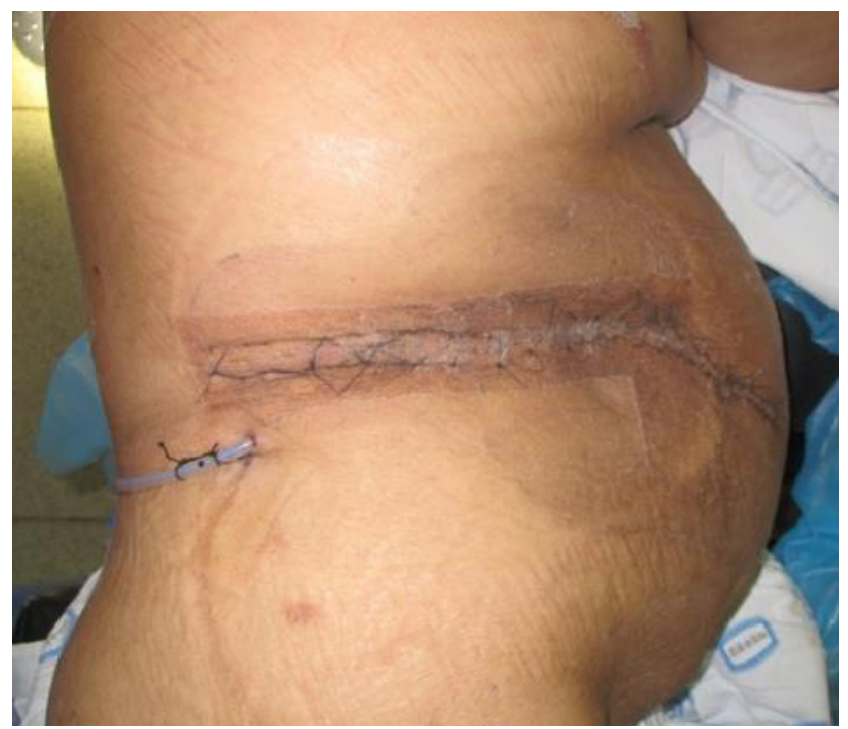

Fig. 5. Post-debridement and flap coverage state. Improved wound condition on day 13 after intravenous antibiotics, surgical debridement, and local flap coverage.

\section{Discussion}

Although a relatively safe and effective procedure in treating biliary obstruction, PTBD has been reported to have a $3 \%$ to $10 \%$ complication rate. Documented problems vary from access site discomfort to fatal vascular insufficiency, most of them being self-limiting. The spectrum of PTBD complications is vast and divided into access-related, nonvascular, vascular or bleeding, catheter-related, and stent-related complications. Most abscess-related complications of PTBD are intraperitoneal abscesses, usually caused by ascending infection from the normal flora of the gut. Mortality rates have been reported to range from $0.1 \%$ to $0.8 \%$ [7]. Extraperitoneal infections usually involve mild to moderate infection of the PTBD insertion site, which are usually treated with empirical intravenous antibiotic administration. Extraperitoneal infections are also mostly caused by ascending infections via the catheter from gut or bile leakage [8]. Rarely have there been reports on extensive extraperitoneal infections. This is probably because the patient is usually receiving antibiotic treatment, and any bile leakage is drained along the catheter tract. Infections that extend to necrotizing fasciitis have not been reported, and the authors believe that the diabetes and ESRD that this patient suffered from made her more vulnerable to a fulminant infection.

Necrotizing fasciitis is a progressive, polymicrobial, and sometimes life-threatening infection of the muscular fascia with necrosis. Non-necrotizing infections invade subcutane- 
ous tissue as well as skin, and may be treated by antibiotics alone. Necrotizing infections, however, involve muscle and fascia as well as skin and subcutaneous tissue, and require emergent surgical intervention. A scoring system based on laboratory findings [5] has been developed to differentiate this condition from simple cellulitis as seen in Table 1. Diagnosis is based on history taking, physical examination, and imaging studies such as CT or magnetic resonance imaging. Risk factors include diabetes, vascular disease, immune compromise, and recent history of operation. The mainstays of treatment consist of adequate fluid resuscitation with electrolyte correction and aggressive application of broad-spectrum antibiotics coverage following radical debridement of all involved tissue. Necrotizing fasciitis caused by enterocutaneous causes has special clinical characteristics in that the source of infection from the digestive system contains digestive enzymes as well as multibacterial contents. For this reason, necrotizing fasciitis with enterocutaneous sources may have a more rapid progress and greater severity [9]. Thus, surgical debridement is of even more importance in such enterocutaneous necrotizing fasciitis, in order to irrigate and remove such enzymatic materials as well as expose the anaerobic environment [10]. In cases involved with PTBD, treatment of the underlying biliary tract infection or problem should be simultaneously undertaken with aggressive necrotizing fasciitis treatment, in order to eliminate the cause of infection as well as the result.

\section{Conflict of interest}

No potential conflicts of interest relevant to this article are reported.

\section{Acknowledgments}

$\begin{array}{ll}\text { Jangyoun Choi } & \text { https://orcid.org/0000-0002-5165-8414 } \\ \text { Jin Tae Cho } & \text { https://orcid.org/0000-0001-7052-9418 } \\ \text { Jong Yun Choi } & \text { https://orcid.org/0000-0002-1164-4499 }\end{array}$

Sung-No Jung https://orcid.org/0000-0002-0419-4717

Bommie Florence Seo https://orcid.org/0000-0002-6907-5962

\section{References}

1. Covey AM, Brown KT. Percutaneous transhepatic biliary drainage. Tech Vasc Interv Radiol 2008;11:14-20.

2. Duan F, Cui L, Bai Y, et al. Comparison of efficacy and complications of endoscopic and percutaneous biliary drainage in malignant obstructive jaundice: a systematic review and metaanalysis. Cancer Imaging 2017;17:27.

3. Saad WE, Wallace MJ, Wojak JC, et al. Quality improvement guidelines for percutaneous transhepatic cholangiography, biliary drainage, and percutaneous cholecystostomy. J Vasc Interv Radiol 2010;21:789-95.

4. Chen CW, Hsiao CW, Wu CC, et al. Necrotizing fasciitis due to acute perforated appendicitis: case report. J Emerg Med 2010; 39:178-80.

5. Wong CH, Khin LW, Heng KS, et al. The LRINEC (Laboratory Risk Indicator for Necrotizing Fasciitis) score: a tool for distinguishing necrotizing fasciitis from other soft tissue infections. Crit Care Med 2004;32:1535-41.

6. Ahmed M. Acute cholangitis: an update. World J Gastrointest Pathophysiol 2018;9:1-7.

7. Venkatanarasimha N, Damodharan K, Gogna A, et al. Diagnosis and management of complications from percutaneous biliary tract interventions. Radiographics 2017;37:665-80.

8. Weber A, Gaa J, Rosca B, et al. Complications of percutaneous transhepatic biliary drainage in patients with dilated and nondilated intrahepatic bile ducts. Eur J Radiol 2009;72:412-7.

9. Wong $\mathrm{CH}$, Chang $\mathrm{HC}$, Pasupathy S, et al. Necrotizing fasciitis: clinical presentation, microbiology, and determinants of mortality. J Bone Joint Surg Am 2003;85:1454-60.

10. Gu GL, Wang L, Wei XM, et al. Necrotizing fasciitis secondary to enterocutaneous fistula: three case reports. World J Gastroenterol 2014;20:7988-92. 